\title{
Nonlinear unbalanced Bessel beams in the collapse of Gaussian beams arrested by nonlinear losses
}

\author{
Miguel A. Porras, Alberto Parola \\ Departamento de Física Aplicada, Universidad Politécnica de Madrid, Rios Rosas 21, ES-28003, Spain \\ CNISM and Department of Physics, University of Insubria, Via Valleggio 11, IT-22100 Como, Italy
}

\begin{abstract}
Collapse of a Gaussian beam in self-focusing Kerr media arrested by nonlinear losses may lead to the spontaneous formation of a quasi-stationary nonlinear unbalanced Bessel beam with finite energy, which can propagate without significant distortion over tens of diffraction lengths, and without peak intensity attenuation while the beam power is drastically diminishing.
\end{abstract}

Nonlinear unbalanced Bessel beams (NL-UBB) have been recently described as the only possible type of light beam that can propagate without distortion and attenuation in a medium with nonlinear mechanisms of dissipation of energy. Contrary to spatial solitons, NL-UBB transport infinite power, which allows for a continuous transversal energy flux from the beam periphery towards its nonlinear center, refuelling the power absorbed in this region during propagation. The idea of NL-UBB has been extended to Bose-Einstein condensates, where similar long-lived structures are supported by the dissipation provided by three-body inelastic collisions.

A spatial dynamics showing the transformation of a Bessel beam into a NL-UBB in a Kerr medium with nonlinear losses (NLL) has been observed . More importantly, the transformation of an ultrashort pulsed Bessel beam into a pulsed NL-UBB may take place without the excitation of any temporal dynamics, [4] as the spectral broadening and time splitting that would occur in the Bessel beam in absence of NLL. These NL-UBB stabilized by NLL have indeed been observed in recent experiments.

The robustness of the NL-UBB suggests that it may act as an attractor of the Kerr dynamics with NLL over a broader space of input conditions. We report here on numerical simulations of the propagation of an input Gaussian beam showing the spontaneous formation of a quasistationary light beam with most of relevant features of a NL-UBB. The different regimes of propagation of a Gaussian beam in a nonlinear medium with NLL were identified NL-UBBs are seen here to emerge in case of strong self-focusing stopped by weak NLL. Contrarily to a Bessel beam, the Gaussian beam carries finite power. As a consequence, only the nonlinear core of the NL-UBB is formed, whose stationarity is seen to be sustained by an inward energy flux from the beam periphery. However, since the available power is finite and decreasing, stationarity lasts after a propagation distance, which is, nevertheless, much larger than the diffraction length associated to the nonlinear core. Our monochromatic approach is expected to describe also experiments with short pulses insofar NLL is the mechanism stopping collapse, and can be assumed to quench any significant temporal dynamics afterwards.
The nonlinear Schrödinger equation (NLSE),

$$
\partial_{z} A=\frac{i}{2 k} \frac{1}{r} \partial_{r}\left(r \partial_{r} A\right)+i \frac{k n_{2}}{n}|A|^{2} A-\frac{\beta^{(K)}}{2}|A|^{2 K-2} A,
$$

is assumed to describe the propagation of a light beam $E=A(r, z) \exp (-i \omega t+i k z)$ with revolution symmetry along the $z$ axis in a medium with Kerr nonlinearity (linear and nonlinear refraction indexes $n$ and $n_{2}>0$, respectively) and multi-photon absorption (coefficient $\left.\beta^{(K)}>0, K=2,3 \ldots\right)$. For the incident Gaussian beam $A(r, 0)=\sqrt{I_{0}} \exp \left(-r^{2} / s_{0}^{2}\right)$ of peak intensity $I_{0}$ and width $s_{0}$, we introduce dimensionless amplitude $\tilde{A}=A / \sqrt{I_{0}}$, radial coordinate $\rho=r / s_{0}$, and propagation distance $\xi=z / k s_{0}^{2}$, to write (1) as

$$
\partial_{\xi} \tilde{A}=\frac{i}{2} \frac{1}{\rho} \partial_{\rho}\left(\rho \partial_{\rho} \tilde{A}\right)+i g|\tilde{A}|^{2} \tilde{A}-\gamma|\tilde{A}|^{2 K-2} \tilde{A},
$$
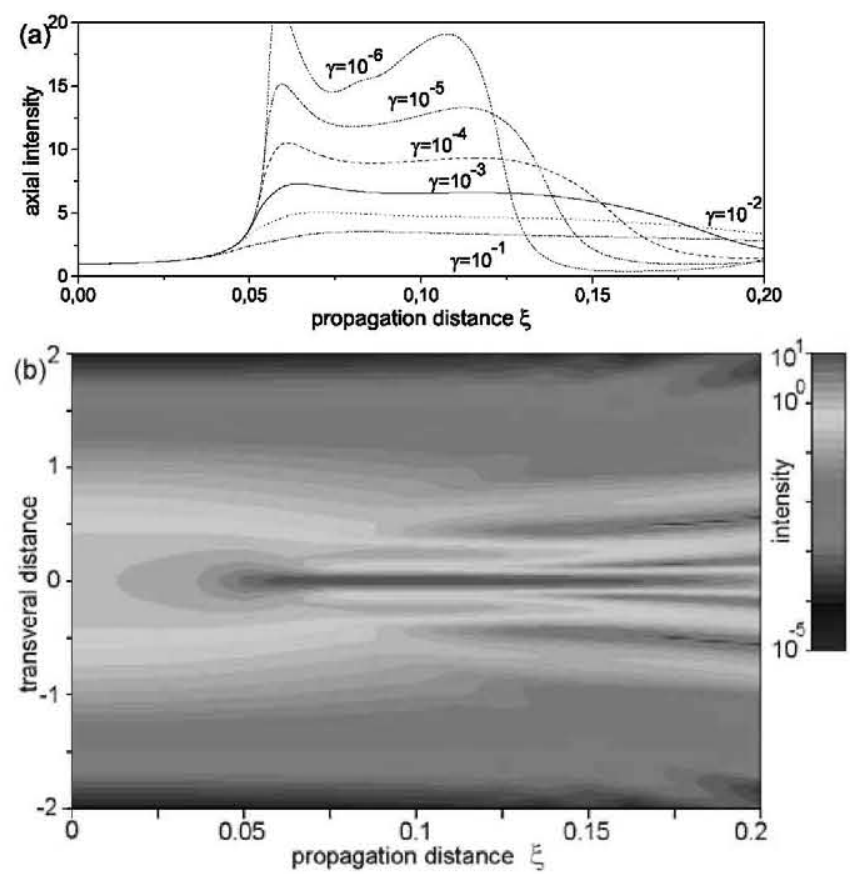

FIG. 1: (a) On-axis intensity $|\tilde{A}(0, \xi)|^{2}$ for input Gaussian with $g=60$ and increasing $\gamma(K=8)$. (b) Intensity $|\tilde{A}(\rho, \xi)|^{2}$ for $g=60$ and $\gamma=10^{-3}(K=8)$ (in logarithmic scale). 


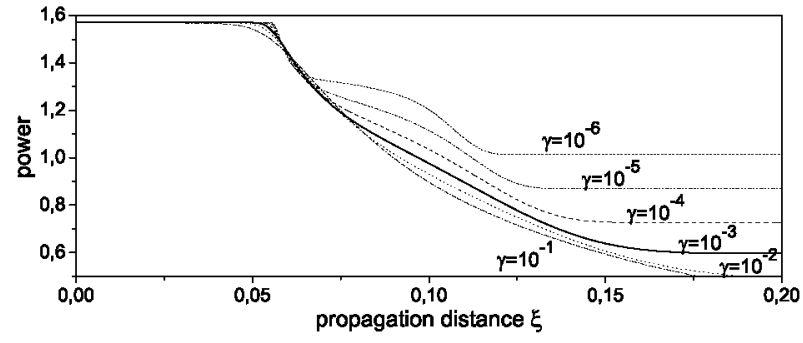

FIG. 2: Beam power $2 \pi \int_{0}^{\infty} d \rho \rho|\tilde{A}(\rho, \xi)|^{2}$ as a function of propagation distance $\xi$ for $g=60$ and increasing $\gamma(K=8)$.

and the initial condition as $\tilde{A}(\rho, 0)=\exp \left(-\rho^{2}\right)$, where $g=k^{2} n_{2} I_{0} s_{0}^{2} / n$ and $\gamma=\beta^{(K)} I_{0}^{K-1} k s_{0}^{2} / 2$ measure the strengths of Kerr nonlinearity and of NLL in the input Gaussian beam.

Without NLL, the Gaussian beam collapses if $g>$ $g_{0} \simeq 3.79$. [6] Figure 1 (a) displays the peak intensity $I=|\tilde{A}(0, \xi)|^{2}$ along $\xi$, numerically evaluated from (2) with $g=60 \gg g_{0}$, where the strong collapse is arrested by different amounts of NLL $\gamma(K=8)$. Similar trend is observed for other values of $g, \gamma$ and $K$ such that the characteristic lengths $\xi_{\mathrm{SF}}=1 /(2 \sqrt{g}), \xi_{\mathrm{D}}=1 / 2$, and $\xi_{\mathrm{NLL}}=\left(2^{K-1}-1\right) /[2 \gamma(K-1)]$ of self-focusing, diffraction and NLL of the input Gaussian

satisfy $\xi_{\mathrm{SF}} \ll \xi_{\mathrm{D}} \ll \xi_{\mathrm{NLL}}$. Under these conditions NLL are negligible in the fast self-focusing, becoming important only in the collapse region, where they prevent the formation of a singularity. With increasing $\gamma$, multiple collapse events disappear. Ripples in the peak intensity $I$ beyond the sole collapse become gradually smoother, and an axial region of nearly constant, high intensity (between $\gamma=10^{-3}$ and $10^{-4}$ for $g=60$ ), or slowly decreasing intensity (for higher $\gamma$ ) is formed. A rough estimate of the peak intensities $I$ (in units of the input intensity $I_{0}$ ) involved in this region is provided by the simple formula $I \approx(g / \gamma)^{1 /(K-2)}$ (e.g., $I=19.8,6.3$ and 2.9 for $\gamma=10^{-6}, 10^{-3}$ and $10^{-1}$, respectively) which is obtained, remarkably, by equating the strength $g I$ of the Kerr nonlinearity (for a field of intensity $I$ ) to the strength $\gamma I^{K-1}$ of NLL.

The transversal profile after collapse features a central spike surrounded by one or more rings, as seen in Fig. 1(b). The width of the central spike can be characterized by $1 / \sqrt{2 g I}$, remaining constant as long as $I$ is constant. Fig. 1(b) corresponds to the case of flattest peak intensity $I\left(\gamma=10^{-3}\right)$, and evidences that the inner portion of the transversal profile (the central spike and one ring) remains quasi-stationary in the region of flat peak intensity, while outer rings are seen to spread due to diffraction. The axial length $\simeq 0.05$ of this region is estimated to be about 17 times the diffraction length $s^{2} / 2 \simeq 0.003$ associated to the width $s \simeq 0.077\left(1 / e^{2}\right.$ intensity decay) of the central spike.
Figure (2) shows that these properties of stationarity are accompanied, paradoxically, by a drastic diminution of the beam power due to NLL, which are greatly enhanced in this region of high intensity. Being nonlinear, absorption takes place mainly in the inner portion of the beam, just that remaining stationary. Along the region of flat peak intensity $\left(\gamma=10^{-3}\right)$, for instance, about one half of the beam power is absorbed.

Stationarity with non-negligible NLL is the distinguishing property of NL-UBBs. These are solutions to the NLSE $(2)$ of the form $\tilde{A}(\rho, \xi)=a(\rho) \exp [i \phi(\rho)-i \delta \xi]$, with $\delta>0$, whose stationary real amplitude $a(\rho)>0$ and phase $\phi(\rho)$ satisfy, from (2),

$$
\begin{array}{r}
\frac{1}{\rho} \frac{d}{d \rho}\left(\rho \frac{d a}{d \rho}\right)-a\left(\frac{d \phi}{d \rho}\right)^{2}+2 \delta a+2 g a^{3}=0 \\
-2 \pi \rho a^{2} \frac{d \phi}{d \rho}=2 \gamma 2 \pi \int_{0}^{\rho} d \rho \rho a^{2 K} .
\end{array}
$$

Eq. (4) is a refilling condition, establishing that the nonlinear power loss (per unit propagation length) in any disk of radius $\rho$ must equal to the power entering into it across its boundary (per unit length) for stationarity to be possible. Given $g, \gamma$ and $K, \mathrm{NL}-$ UBBs solutions to (3) and (4) exist with any peak intensity $I=a^{2}(0)$ and axial wave number shift $\delta \gtrsim$ $\min \left\{0,2 \eta_{K} \gamma I^{K-1}-g I\right\}$, as illustrated in Fig. 3(a), where $\eta_{K}=1.67,0.27,0.19,0.16,0.14,0.12,0.11, \ldots$ for $K=2,3, \ldots$ Far from its nonlinear core, the NL-UBB approaches zero as $\rho \rightarrow \infty$ as an unbalanced Bessel beam, a generalization of the Bessel beam with unequal amplitudes of its inward and outward Hänkel beam components, and that carries, as Bessel beams, infinite power. [1] Figure 3(b) shows radial intensity profiles and inward radial energy fluxes of NL-UBBs with fixed intensity $I$ and decreasing $\delta$, evaluated from Eqs. (3) and (4). At fixed $g, \gamma$ and $K$, NL-UBB profiles depend on the two parameters $I$ and $\delta$, but as $\delta$ diminishes down to $\delta=0^{+}$at fixed $I$ [arrow in Fig. 3(a)], a limiting profile determined
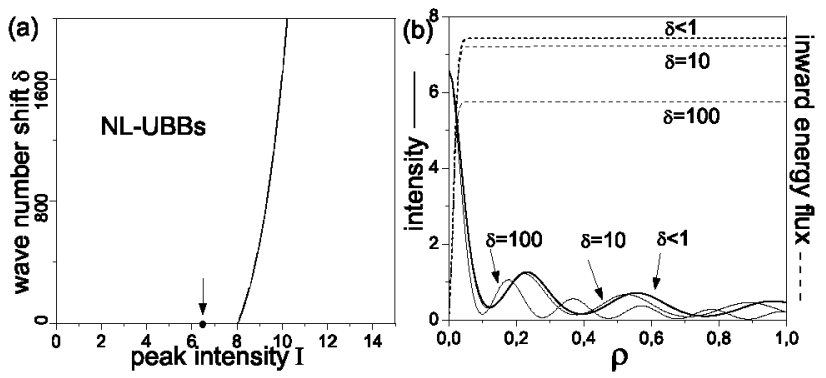

FIG. 3: (a) For $g=60, \gamma=10^{-3}$ and $K=8$, region of existence of NL-UBBs in their parameter space $(I, \delta)$. (b) Radial intensity profiles $a^{2}(\rho)$ and inward radial energy fluxes $-2 \pi \rho a^{2}(\rho) d \phi(\rho) / d \rho$ for NL-UBBs with fixed $I=6.575$ and decreasing $\delta$ down to $0^{+}$, as indicated by the arrow in (a)]. 


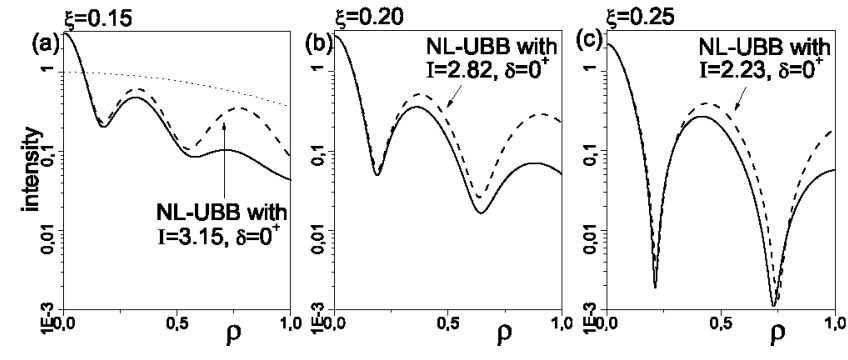

FIG. 4: For $g=60, \gamma=10^{-1}, K=8$ and input Gaussian (dotted curve), radial intensity profiles of the propagated field at increasing distances beyond collapse (solid curves), and radial intensity profiles of NL-UBBs with same peak intensities $I=3.15,2.82,2.23$ as those of the propagated field at each distance, and $\delta=0^{+}$(dashed curves).

only by $I$ is reached [solid thick curve in Fig. 3(b)].

Coming back to the collapse of the Gaussian beam, the slow evolution after collapse is interpretable as an adiabatic sequence of NL-UBBs with slowly varying peak intensity $I$ and $\delta=0^{+}$. Figure 4 shows, for $g=60$, $\gamma=10^{-1}$ [lowest curve in Fig. 1(a)], that the inner part of the radial profile at each distance $\xi$, comprising the central maximum and about one ring, fits well to that of the NL-UBBs with $\delta=0^{+}$and with $I$ equal to that of the propagated field. A truly stationary NL-UBB cannot be fully formed because of the finite power available, which forces the slow evolution in the parameter space of NLUBBs, with intensities about $I=(g / \gamma)^{1 /(K-2)}=2.9$. The fact that NL-UBBs with $\delta=0^{+}$are always formed can be understood by thinking on the collapsing beam as a bundle of rays at different angles down to zero, or equivalently, different Bessel beams with decreasing $\delta$ (or cone angle). Each Bessel beam with $\delta \neq 0$ forms indeed its own NL-UBB with same $\delta \neq 0$ in the nonlinear medium, as demonstrated in [3], but only those with $\delta \rightarrow 0$, whose radial profile is stationary against a change of $\delta$, emerge.

Figure 5(a) is similar to Fig. 4 but for $\gamma=10^{-3}$ with flatter peak intensity [see also Figs. 1(a) and (b)]. The mechanism of energy refilling that sustains quasi-stationarity is evidenced in Fig. 5(b). Writing $\tilde{A}(\rho, \xi)=a(\rho, \xi) \exp [i \varphi(\rho, \xi)]$, where $a>0$ and $\varphi$ are the real amplitude and phase, the NLSE (2) yields

$$
\frac{d}{d \xi} 2 \pi \int_{0}^{\rho} d \rho \rho a^{2}=-2 \pi \rho a^{2} \partial_{\rho} \varphi-2 \gamma 2 \pi \int_{0}^{\rho} d \rho \rho a^{2 K}
$$

or $d P_{\rho} / d \xi=F_{\rho}-N_{\rho}$ for short, which generalizes (4) to a non-stationary field. Relation (5) expresses that the variation of the power $P_{\rho}$ in a disk of radius $\rho$ can be due to an inward energy flux $F_{\rho}$ across its boundary, and to the nonlinear power loss $N_{\rho}$ within it. For NL-UBBs, $d P_{\rho} / d \xi=0$, and (5) reduces to the refilling condition (4), or $F_{\rho}=N_{\rho}$. In particular, the total nonlinear power loss $N_{\infty}$ is compensated in the NL-UBB by a constant energy flux $F_{\infty}$ coming from infinity [horizontal asymptota labelled as NL-UBB in Fig. 5(b)], this being made feasible by the infinite power in the NL-UBB. In the collapsed Gaussian beam, instead, the total nonlinear power loss $N_{\infty}$ (gray horizontal asymptotas) is not compensated by any inward flux from infinity, since $F_{\infty}=0$ (black curves approaching zero), and the beam power diminishes according to $d P_{\infty} / d \xi=N_{\infty}$ (as in Fig. 2). Nevertheless, the collapsed Gaussian beam is seen in Fig. 5(b) to develop an inward energy flux in its inner portion such that the refilling condition $F_{\rho}=N_{\rho}$ characteristic of the NLUBBs, and hence $d P_{\rho} / d \xi=0$, are approximately satisfied where the profile fits the NLL-UBB.

The sequence of NL-UBBs is also observed after collapse with smaller NLL $\left(\gamma<10^{-6}\right.$ for $\left.g=60\right)$, even after secondary collapse events. Due to the faster variation of $I$, however, a narrower portion of the NL-UBB profiles (sometimes only the central spike) gets formed.

We can then conclude that NL-UBBs tend to be formed spontaneously in Kerr media with NLL, not only from a beam with infinite power as a Bessel beam, but also from a Gaussian beam that experiences strong selffocusing. Due to the finite power available, the attracting NL-UBB is never fully reached, and is hence hard to be characterized. Nevertheless, the numerical simulations indicate that the system seeks the NL-UBB with $\delta=0^{+}$and a peak intensity $I \approx(g / \gamma)^{1 /(K-2)}$ balancing the strengths of Kerr nonlinearity and NLL.

M. A. Porras, A. Parola, D. Faccio, A. Dubietis, and P. Di Trapani, Phys. Rev. Lett. 93, 153902 (2004).

A. Alexandrescu, and V. M. Pérez-García, Phys. Rev. A 73, $053610(2006)$.

P. Polesana, A. Dubietis, M. A. Porras, E. Kucinskas, D. Faccio, A. Couairon, and P. Di Trapani, Phys. Rev. E 73, $056612(2006)$.
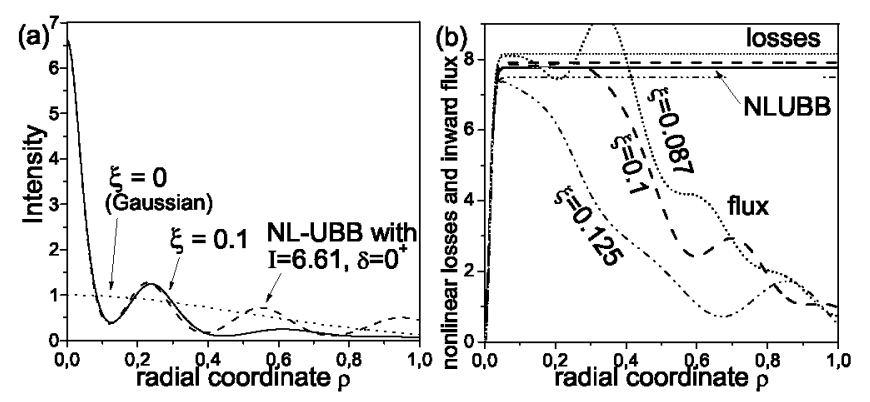

FIG. 5: For $g=60, \gamma=10^{-3}, K=8$, (a) radial intensity profiles of input Gaussian, propagated field and NL-UBB with same peak intensity $I=6.61$ as the propagated field, and $\delta=0^{+}$. (b) Inward radial energy flux $F_{\rho}$ (black curves) and NLL $N_{\rho}$ (gray curves) at increasing propagation distances $\xi$. For the NL-UBB, inward flux and NLL are equal at any $\rho$. 
P. Polesana, A. Couairon, D. Faccio, A. Parola, M. A. Porras, A. Dubietis, A. Piskarskas, and P. Di Trapani, Phys. Rev. Lett. 99, 223902 (2007).

S. Polyakov, F. Yashino, and G. Stegeman, J. Opt. Soc. Am. B 18, 1891 (2001).
[6] This quantity corresponds to $2 \alpha, \alpha=1.8962$ given in G. Fibich and A. Gaeta, Opt. Lett. 25, 335 (2000).

[7] B. J. LeMesurier, Phyica D 138, 334 (2000). 